\title{
Conservazione e valorizzazione dei sistemi fortificati ridotti allo stato di rudere in Val Tanaro (Piemonte)
}

\author{
Conservation and enhancement of ruined fortified systems in Tanaro Valley (Piedmont)
}

\section{Emanuele Romeo}

Politecnico di Torino, Turin, Italy, emanuele.romeo@polito.it

\begin{abstract}
The contribution reports on a research concerning the preparatory investigation of some fortified systems of Tanaro Valley (Piedmont) in view of their restoration. The research highlighted the strong connection between the castles and the memory of events and characters that were linked to these architectures; the relationship between landscape and ruins that, today, represents a potential resource for the territory; the willingness of local authorities to improve knowledge, protection and enhancement of this heritage; the need to investigate theoretical and methodological issues and define the proper procedures for the conservation, restoration, safeguard, maintenance and management of a widespread and vulnerable heritage. The study, taking advantage of interdisciplinary contributions, used innovative instruments for the survey and monitoring of this heritage, as well as graphic and digital restitution techniques to propose to the scientific community -and communicate to the native population- the interventions of restoration, conservation, enhancement and management of these fortified systems. The research demonstrates that particular attention has to be paid to these less-known historical architectures that can however contribute, as effectively as the most famous and eye-catching fortification systems, to the political and social development and to the historical identity whose recognition, preservation and valorization are strongly hoped for.
\end{abstract}

Keywords: Fortified systems, conservation, enhancement.

\section{Il valore delle rovine nel paesaggio}

Il tema della tutela e della valorizzazione dei contesti territoriali antropizzati assume oggi un ruolo centrale nel più ampio quadro del paesaggio culturale, dove ruderi (sia di età classica, sia medievali), architetture storicamente più recenti e vegetazione assurgono a sistema unitario tutelato, restaurato e conservato grazie al riconoscimento di valore attribuito ai singoli frammenti o ai più complessi sistemi di rovine. In Val Tanaro $(\mathrm{CN})$, il sistema di fortificazioni e di castelli risponde ai requisiti di paesaggio culturale, comprendendo rovine di età medievale e architetture fortificate più recenti, e pertanto merita di essere conservato e valorizzato. Tuttavia, prima di proporre alcune linee guida sul restauro, è necessario comprendere le matrici storiche del dibattito grazie al quale, oggi, è possibile parlare di paesaggio archeologico e culturale. Da un lato, la rovina (ri-contestualizzata o artificialmente riprodotta) è risorsa aggiuntiva del tessuto vegetale, con tutti i rimandi al valore di antichità; dall'altra è la vegetazione che, unitamente al plusvalore della natura, aumenta il valore stesso della memoria storica espressa dal rudere. Natu- 
ra e vegetazione esprimono, quindi, un'endiadi che trova, nelle attuali esperienze di valorizzazione del paesaggio, un territorio per nuovi confronti progettuali (Tosco, 2007). Il ruolo che la natura da sempre ha assunto, come luogo del ricordo di avvenimenti storici -anche con la presenza di ruderi che ne accentuano il valore di memoria- è noto, ed è stato analizzato dalla letteratura critica (Romeo 2004). Infatti, parlando del rapporto che intercorre tra le rovine di età classica e il paesaggio, è necessario citare Plinio, il quale, auspicando il rispetto di alcuni contesti naturali, pone la conservazione degli elementi naturali - soprattutto di quelli che sono stati scenario di eventi storici- come alternativa rispetto al loro sfruttamento: "I nostri padri consideravano come un miracolo il passaggio di Annibale attraverso le Alpi, e poi quello dei Cimbri; quelle stesse Alpi ora vengono tagliate in mille specie di marmi, e i promontori vengono aperti al mare, e il mondo si livella. Noi asportiamo quelle barriere che erano costruite per naturale confine tra gente e gente, e si fabbricano navi per caricare i marmi, e le cime dei monti vengono così trasportate qua e là sui flutti, che sono il più terribile elemento della natura" (Plinio, 2001). Ancora più attente sono le considerazioni di Pausania, per il quale i ruderi assumono valore, e vanno per questo conservati, solo se legati alla natura in un paesaggio dove aspetti cultuali, sociali, religiosi, storici si fondono, assegnando ai luoghi valore di memoria. È in Arcadia che il Periegeta apprezza le rovine e il paesaggio. Insieme, essi conferiscono alla regione il ruolo di luogo della memoria storica della Grecia, acquistando valore d'uso grazie alla frequentazione dei siti, ed al legame che esiste tra la storia e l'uso attuale del paesaggio e delle rovine: tra i ruderi del tempio di Apollo Pizio resta " $[. .$.$] un$ grande altare di marmo bianco. Qui ancor oggi i Feneati sacrificano ad Apollo e ad Artemide" (Pausania, 2001). I miti antichi e gli dei ancestrali, i tradizionali culti e i più importanti eventi storici, vivi nella memoria attraverso le rovine, sono compenetrati nel paesaggio, visto come potenziale punto di partenza per riflessioni sull'utilizzo culturale del territorio: la funzione, nel passato, delle sorgenti, dei boschi, delle pianure, delle montagne, delle zone paludose e di quelle coltivate è testimoniato dalla presenza delle rovine. L'importanza del loro rispetto e della loro conservazione è dato dal valore di attualità che ancora rivestono. Ma il ruolo, nella storia, del patrimonio ruderizzato, non solo paesaggistico ma anche urbano, è sottolineato da Victor Hugo che nella lettera del 1883 (27 luglio) inviata al Presidente del Consiglio Municipale, in difesa dell'arena di Lutèce a Parigi, ribadisce l'importanza della conservazione dell'anfiteatro parigino in quanto monumento utile affinché si potesse, idealmente, collegare la città del futuro con la città del passato, attribuendo al monumento gallo-romano un significativo valore storico: "Il n'est pas possible que Paris, la ville de l'avenir, renonce à la preuve vivante qu'elle a été la ville du passé. Le passé amène l'avenir. Les arènes sont l'antique marque de la grande ville. Elles sont un monument unique. Le conseil municipal qui les détruirait se détruirait en quelque sorte lui-même. Conservez les arènes de Lutèce. Conservez-les à tout prix. Vous ferez une action utile, et, ce qui vaut mieux, vous donnerez un grand exemple" (Romeo, 2012, p. 14). Secondo il letterato, la conservazione del monumento sarebbe stata di esempio per future azioni di salvaguardia del patrimonio francese, sebbene, in quegli anni, tutto ciò che non concorreva alla progettazione della nuova città apparisse inutile. L'arena parigina diventò così il simbolo del passato nella città futura, assumendo il ruolo di elemento catalizzatore delle attività sociali e culturali di un intero quartiere. Tuttavia, a livello europeo, vi erano, allora, posizioni contrastanti circa la conservazione del patrimonio storico, ritenuto spesso inutile. La dimostrazione, in territorio italiano, è rappresentata dalla nota posizione dei Futuristi, i quali disprezzavano i documenti del passato tanto da suggerirne la distruzione, sia nei contesti urbani, sia nel paesaggio. In tal senso si esprime Umberto Boccioni, che auspica una maggiore trasformazione del territorio storico a fronte di nuove esigenze della contemporaneità: "Non possiamo pensare senza disgusto e compassione che esistono società per la conservazione del paesaggio. [...] Imbecilli! Conservare che cosa? Ma i paesaggi che ora si vogliono conservare non esistono oggi sul posto e in virtù di altri di- 
strutti o trasformati? Imbecilli! Come se non fosse infinitamente sublime lo sconvolgere che fa l'uomo sotto la spinta della ricerca e della creazione, l'aprir strade, colmare laghi, sommergere isole, lanciare dighe, livellare, squarciare, forare, sfondare, innalzare per questa divina inquietudine che ci spara nel futuro?" (Zevi, 1994). La visione utilitaristica del territorio, luogo per nuove attività produttive ed economiche, esclude dunque la possibilità di una qualche utilità, sia pur solo culturale, del patrimonio storico. A ciò si oppose Gino Chierici che, negli anni Trenta del XX secolo, propose e realizzò la conservazione dell'Abbazia di san Galgano, come rudere medievale inserito nel paesaggio senese. $\mathrm{Al}$ valore di utilità, che Gustavo Giovannoni attribuisce al monumento solo se ricostruito, Chierici antepone l'utilità culturale della rovina. Ancora oggi San Galgano rappresenta un caso emblematico, in cui il rudere assume valore d'uso e di attualità: utilizzato come suggestivo scenario per manifestazioni legate alla cultura e all' arte, è esempio di come una rovina possa essere educativa per ciò che concerne il rapporto tra attività umane e paesaggio. Tali teorie, nell'Italia postunitaria, si erano in parte già concretizzate attraverso l'interesse nei confronti di contesti territoriali in cui la presenza di ruderi contribuiva ad accentuare quel valore di "bellezza naturale", necessario perché alcuni ambiti potessero essere tutelati dalle leggi nazionali di salvaguardia. A tal proposito si possono citare, per restare in territorio piemontese e valdostano, le azioni di tutela e i primi tentativi di conservazione del Plan de Jupiter (presso il Gran San Bernardo) e dell'Arco di Donnaz in Valle d'Aosta; gli studi di D'Andrade, Assandria e Vacchetta a Susa, Acqui Terme, Libarna e Bene Vagienna. In questi casi, parlando del rapporto che intercorre tra rovine e contesto paesaggistico, si auspicava la conservazione degli elementi naturali, soprattutto di quelli che furono scenario di eventi storici. In tal modo l'Italia dimostrò di allinearsi alle politiche di tutela di altri Paesi europei, che ormai da tempo avevano fatto delle rovine gli strumenti più efficaci per comunicare, soprattutto al pubblico meno colto e attraverso tracce visibili e tangibili, la storia del loro territorio: la rovina era considerata, al pari delle altre manifestazioni architettoniche, utile strumento didattico e divulgativo. Si pensi alla tutela dei complessi monastici in Inghilterra; al sistema dei castelli in rovina dei paesi sassoni; a quelli francesi sopravvissuti alla Rivoluzione (Woodward, 2008). Il dibattito post-bellico, inoltre, fece emergere le difficoltà di coloro i quali furono, a quel tempo, chiamati a ricostruire contesti urbani devastati dal secondo conflitto mondiale. Cosa era urgente, nonché utile, costruire ex novo, ripristinare o conservare? E quanto, invece, ritenuto inutile per la crescita economica, poteva essere demolito nelle città o considerato marginale nelle strategie di pianificazione territoriale? Gli esiti di tale dibattito sono ben noti, al punto che, tra gli anni Sessanta e Settanta, diventò necessario affermare l'importanza del patrimonio culturale in quanto Bene che costituisce testimonianza avente valore di civiltà, secondo la definizione della Commissione Franceschini (Dezzi Bardeschi, 2017). Tuttavia, ancora oggi, si ritiene (da parte di molti che governano il territorio) che la conservazione delle tracce del passato, contenute all'interno di paesaggi e città, sia operazione inutile e insostenibile. Fanno eccezione coloro che "sfruttano" il patrimonio, attribuendo a esso mero valore d'attualità, quell'istanza dalla quale metteva in guardia Alois Riegl: pericolosa, inutile culturalmente, soggetta alle mode, derivante solamente da contingenti "tattiche" turistiche ed economiche (Romeo, 2012).

\section{L'utilità culturale della rovina}

Gli elementi naturali, il paesaggio e le presenze tangibili della storia culturale di un territorio antropizzato meritano tuttavia di essere conservati, e le strategie di valorizzazione sostenibili dovrebbero informare i documenti e le norme sulla tutela del paesaggio e del territorio culturale maggior ragione quando a essi sono legate le tracce della storia, percepibili attraverso la presenza di infrastrutture, siti urbani e monumenti-. Attualmente, la definizione di area o sito archeologico come luogo per la conservazione del patrimonio, perlopiù classico, e più di recente anche medievale, viene sostituita con il concetto di paesaggio archeologico come luogo in cui si incontrano elementi architettonici e naturali; tracce urbane di antiche civiltà e elementi vegetali au- 
toctoni; installazioni funzionali e più recenti sistemazioni attraverso l'uso della materia vegetale. Tuttavia, risulta ancora difficile da attuare la tutela di intere porzioni di territorio, che, non avendo specifiche caratteristiche riconducibili alla definizione di parco, potrebbero essere considerate dei paesaggi a rischio (Chavarria Arnau, 2015). Le difficoltà consistono, spesso, nell'impossibilità di perimetrare tali contesti, in cui gli elementi archeologici e naturali sono strettamente collegati, o nella particolare ubicazione di rovine che, con la loro presenza diffusa, insistono su aree private, connotando, al tempo stesso, contesti ricchi di elementi vegetali autoctoni con particolari caratteristiche idrologiche e geo-morfologiche (Romeo, 2009). In tal senso, buona parte della Val Tanaro può essere considerata paesaggio archeologico, poiché alle rovine di manufatti antichi si affiancano infrastrutture storiche, complessi fortificati isolati, insediamenti urbani di origine medievale e architetture più recenti, nate per migliorare nel tempo lo sviluppo economico e sociale dell'intera valle (Rudiero, 2015). Quali strategie di conservazione e valorizzazione possono proporsi affinché si considerino le rovine nel paesaggio (tracciati stradali, torri, castelli, pievi, abbazie, presidi militari, fortificazioni moderne) come indispensabili e utili testimonianze per la comprensione del territorio e per l'arricchimento delle popolazioni autoctone che ne sono, a vario titolo, detentrici? Quelle per le quali, quanto considerato spesso inutile, perché marginale rispetto alle strategie economiche legate al turismo, diventi utile strumento di formazione intellettuale in grado di valorizzare particolarità, tipicità, diversità della Val Tanaro sulla base delle tracce, dei frammenti e delle rovine del passato. Tuttavia, ciò minerebbe "la logica del profitto", in quanto coinciderebbe esclusi-vamente "con il sapere in sé, indipendentemente dalla frenesia di produrre guadagni immediati o benefici pratici. Certo, molto spesso i musei, [i ruderi] o i siti archeologici possono anche essere fonte di straordinari introiti. Ma la loro esistenza, contrariamente a ciò che alcuni vorrebbero farci credere, non può essere subordinata al successo degli incassi: la vita di un museo, o di uno scavo archeologico, come quella di un archivio o di una biblioteca [o di una rovina nel paesaggio], è un tesoro che la collettività deve gelosamente preservare a ogni costo" (Ordine, 2013). Attualmente, come è noto, l'evoluzione del concetto di paesaggio ha dato vita ad un processo che considera non solo il valore materiale di tale patrimonio, ma anche quello immateriale, legato strettamente alle tradizioni delle popolazioni autoctone, inserendo in tale processo di valorizzazione anche il "valore della rovina" (Scazzosi, 2002). In effetti, da un lato, quest'ultima potrebbe rappresentare utile risorsa aggiuntiva del paesaggio, con tutti i rimandi ai valori culturali che essa possiede; dall'altro, il paesaggio aumenta di valore grazie alla memoria storica espressa dal rudere. Tuttavia il territorio antropizzato continua ad essere considerato come utile scenario per lo sviluppo industriale e per le politiche economiche di molte amministrazioni pubbliche, italiane ed europee.

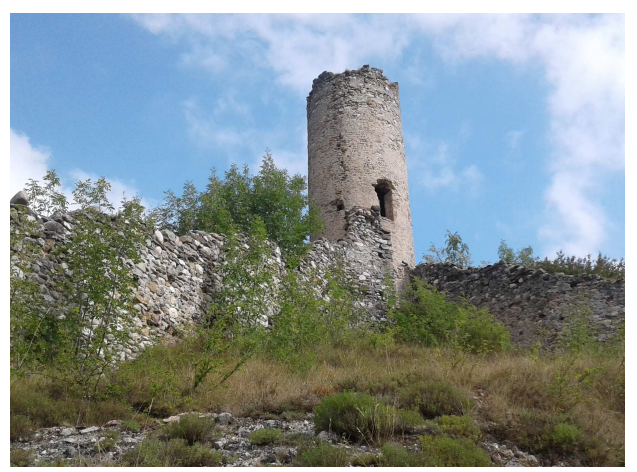

Fig. 1. Bagnasco $(\mathrm{CN})$ : i ruderi della cinta muraria con una delle torri di avvistamento.

$\mathrm{Ne}$ consegue che l'utile risorsa culturale data dall'endiadi indissolubile di rovina e paesaggio diventa inutile elemento, anzi, ostacolo per tali azioni di programmazione di assetto territoriale. Ma se le indicazioni normative e le specifiche leggi di tutela a livello europeo auspicano il rispetto del paesaggio e delle rovine in esso contenute, allora è necessario "inventarsi" un escamotage, attraverso il quale anche l' "inutile" architettura ruderizzata possa risultare "utile" alle suddette politiche di sviluppo economico (Romeo, 2012). Interviene, allora, la cosiddetta "valorizzazione", il cui vero obiettivo non è tanto la tutela e la conservazione di paesaggio e rovine, ma lo sfruttamento di tali beni: ciò ha creato ce- 
sure tra paesaggio e rovina, esponendo a grandi rischi buona parte del patrimonio culturale, compreso quello presente il Piemonte e in special modo in Val Tanaro.

\section{I sistemi fortificati della Val Tanaro}

Il patrimonio architettonico e paesaggistico legato ai sistemi fortificati presenti in tutte le regioni italiane è sempre stato oggetto di attenzione da parte di chi, a vario titolo, si è interessato di salvaguardia, sia dei beni culturali -nell'accezione più generale del termine- sia dei più specifici valori di memoria legati allo sviluppo di quell'aristocrazia che, attraverso il presidio del territorio, ha rafforzato, dal Medioevo sino all'età contemporanea, il proprio predominio politico, sociale, economico.

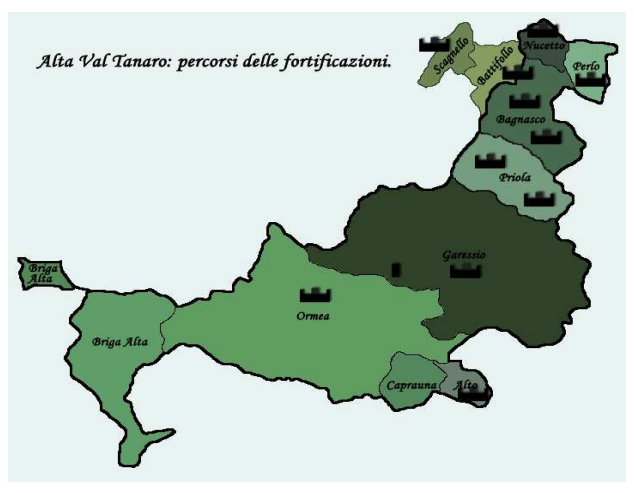

Fig. 2. Mappa dei castelli situati in Val Tanaro (CN).

Tuttavia, esistono realtà meno note che attendono ancora di essere studiate e valorizzate attraverso processi che coinvolgano non solo una ristretta élite culturale, ma anche gli enti locali preposti all'amministrazione di quei territori, per i quali la forte presenza di impianti fortificati, presidi militari o castelli nobiliari, costituisce o concorre a formare il valore di identità territoriale auspicato nelle politiche di salvaguardia nazionali e internazionali. Il contributo vuole presentare la ricerca, in fieri - iniziata qualche anno fa dalla Scuola di Specializzazione in Beni Architettonici e del Paesaggio del Politecnico di Torino - riguardante le indagini propedeutiche agli interventi di restauro di alcuni sistemi fortificati dell'alta Val Tanaro da cui è emerso: il forte legame tra i castelli e la memoria di eventi e personaggi che a queste architetture furono legati; lo stretto rapporto tra paesaggio e rovine, che oggi rappresenta una risorsa per il territorio; la volontà degli enti locali di migliorare la conoscenza, la tutela e la valorizzazione di tale patrimonio; la necessità di approfondire problematiche teorico-metodologiche utili al restauro e alla manutenzione di un patrimonio diffuso sempre più vulnerabile. Lo studio, avvalendosi di contributi interdisciplinari, ha utilizzato strumentazioni innovative per il rilevamento e il monitoraggio di tali beni, nonché tecniche di restituzione grafica e digitale per proporre alla comunità scientifica, e comunicare alla popolazione autoctona, gli interventi di restauro, la conservazione, la valorizzazione e la gestione di questo patrimonio culturale.

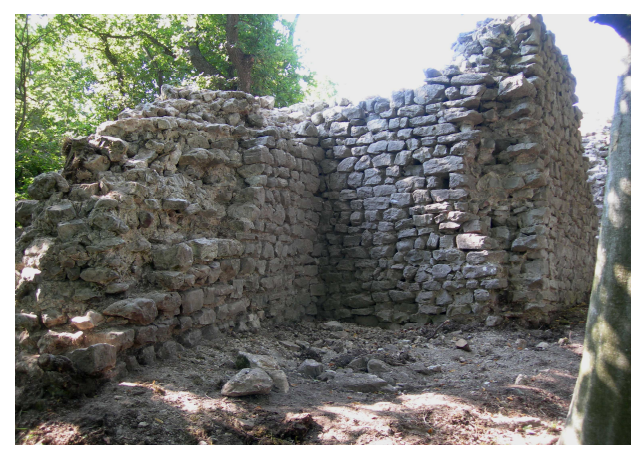

Fig. 3. Bagnasco (CN): la torre del sistema difensivo di Santa Giulitta durante i lavori di pulitura archeologica.

Una particolare attenzione, quindi, alle realtà locali meno note, ma che possono oggi contribuire, parimenti ai casi eclatanti di sistemi di fortificazione, a quello sviluppo politico-sociale e a quell'identità storica che tutti auspichiamo sia riconosciuta. Gli studi condotti presso i siti di Castel Nuovo di Ceva, Nucetto, Bagnasco (in particolar modo, Santa Giulitta), Priola, Ormea, Battifollo, Garessio (solo per citarne alcuni), hanno avuto, principalmente, l'obiettivo di evitare che la valorizzazione di tali contesti paesaggistici venisse fraintesa diventando esclusivamente il volano per azioni solo economiche e turistiche.

Ciò attraverso una serie di sopralluoghi, di analisi cognitive e di indagini sullo stato di conservazione delle architetture fortificate, per impostare un progetto di restauro finalizzato essenzialmen- 
te alla conservazione delle tracce materiali e immateriali e non allo sfruttamento intensivo di questa risorsa culturale (Rudiero, 2015). In effetti, come afferma anche Giovanni Carbonara, si può giungere alla valorizzazione di un bene culturale facendone semplicemente conoscere la storia, incentivandone le opere di manutenzione costante, sensibilizzando enti amministrativi e organismi di tutela, educando la popolazione a saper riconoscere il valore storico e culturale del proprio patrimonio (Carbonara, 2000). Tali siti, a rischio, assieme agli elementi naturali, al paesaggio e alle presenze tangibili della storia di un territorio antropizzato, meritano di essere conservati (Demeglio, 2015).

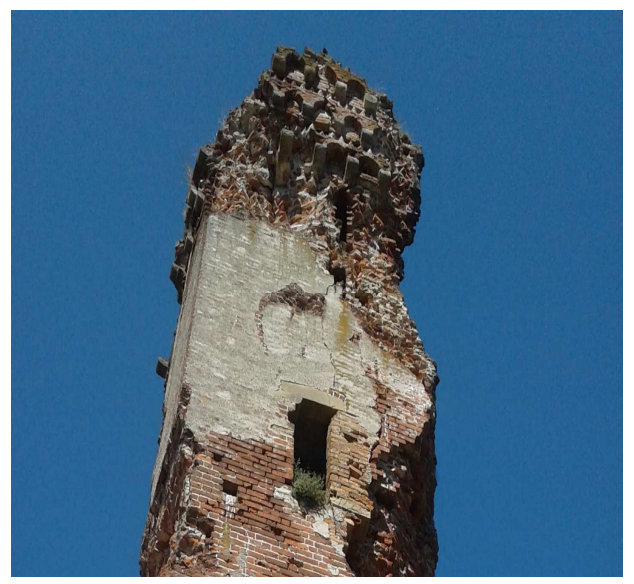

Fig. 4. Battifollo $(\mathrm{CN})$ : i ruderi della torre con i chiari segni di degrado e dissesto delle strutture murarie.

Quindi, sulla base delle criticità riscontrate e in considerazione delle potenzialità dell'intero territorio, è nata l'esigenza di migliorare gli strumenti di conoscenza finalizzati alla conservazione e, in termini di valorizzazione e promozione, è stato necessario mettere a punto strumenti atti a permettere una lettura accompagnata (corretta scientificamente e comprensibile a tutti) di questo patrimonio diffuso, con strategie che siano applicabili a livello locale e nazionale (Rudiero, 2015). Per agevolare la comprensione dei luoghi e indirizzare nello specifico le necessarie analisi, si è pertanto pensato di suddividere il territorio lungo l'arteria stradale principale che, partendo da Ceva e attraversata Ormea conduce al valico del colle di Nava- in due aree specifiche: il sistema di fortificazioni lungo la principale direttrice viaria (Nucetto, Garessio, Bagnasco, Ormea) e i castelli, situati lungo i percorsi viari trasversali (Castel Nuovo di Ceva, Priola, Battifollo, Perlo): complessi, questi ultimi, poco noti $\mathrm{e}$ in condizioni di totale abbandono. In tal senso, sin dai primi sopralluoghi presso i sistemi fortificati, si sono predisposti i siti affinché fossero consentite le prime indagini. In primo luogo, dopo un'attenta ricognizione, si è provveduto alla parziale liberazione dei ruderi dalla vegetazione infestante, cercando di evitare quel processo di desertificazione che spesso connota gli iniziali interventi di restauro sul patrimonio ruderizzato conservato in contesti naturali (Lambrick, 1985). Tale processo ha richiesto la conoscenza delle specie autoctone che andavano conservate e ha previsto una serie di analisi sulla vegetazione ruderale, allo scopo di rimuovere quelle dannose per le strutture in elevato e risparmiare quelle che avrebbero potuto garantire la conservazione del sistema paesaggio-rovina. Si è trattato di individuare (per rimuoverle) le specie arboree e arbustive che danneggiavano con gli apparati radicali le strutture, e quelle le cui radici collaboravano all'efficienza strutturale delle murature fuori terra (per conservarle). Inoltre, alcune specie di licheni avevano garantito la conservazione degli strati più superficiali dei paramenti murari e necessitavano interventi di monitoraggio per verificarne la dannosità e quindi l'eventuale rimozione. A queste indagini sono seguite le operazioni di catalogazione dei frammenti erratici presenti presso le strutture e la lettura attenta dei crolli primari, o l'individuazione delle porzioni di muratura asportate nei secoli a causa dei saccheggi da parte delle popolazioni locali. Ciò ha consentito di poter ipotizzare un quadro fessurativo, individuando lesioni e deformazioni: esse, pur non interessando le strutture principali di quasi tutti i castelli analizzati, sono risultate presenti su estese porzioni di muratura fuori terra, soprattutto nei casi di quelle architetture in cui vi erano state maggiori espoliazioni. Si è, quindi, ipotizzato di conservare le strutture in crollo primario, come testimonianza della "storia statica" del 
complesso e come documento delle tracce sismiche del territorio, pensando, invece, di ricostruire le porzioni di muratura collassate necessarie al ripristino strutturale delle cortine più a rischio. Un ruolo importante hanno poi avuto gli studi per la pubblicizzazione in itinere sia delle fasi conoscitive, sia di quelle esecutive; una sorta di live restoration, con la finalità di rendere partecipe il pubblico (dagli esperti ai non addetti ai lavori) non solo rispetto alle indagini storiche, ma anche rispetto alle scelte metodologiche adottate. Ciò attraverso incontri con i rappresentanti delle amministrazioni locali, con i responsabili delle associazioni culturali e con la popolazione (Rudiero, 2013).

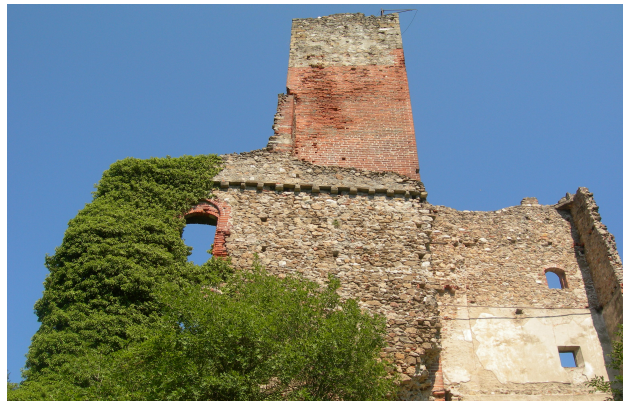

Fig. 5. Nucetto $(\mathrm{CN})$ : le rovine del castello con le varie fasi di ampliamento della struttura fortificata.

\section{Conclusioni}

Un lavoro di equipe, ancora in progress, che ha avuto come obiettivo la conoscenza per la conservazione e la valorizzazione dei castelli della Val Tanaro, attraverso la lettura delle testimonianze architettonico-paesaggistiche, per poter redigere mappe, pubblicare dati storici, proporre ricostruzioni virtuali (dei ruderi o dei sistemi più complessi) che ne evidenzino gli aspetti formali, stilistici e materici; le originarie configurazioni architettoniche; le differenti trasformazioni. Tutto questo per far conoscere una complessa realtà territoriale che -sebbene non sufficientemente rappresentativa di quella "globalizzazione culturale" sempre più condizione indispensabile nelle politiche di salvaguardia- rappresenta pur sempre la cultura e le identità locali. Ciò per soddisfare quanto richiesto dalle normative internazionali $\mathrm{e}$, in particolare, dalla Convenzione europea sul
Paesaggio, in cui, riconoscendo qualità $\mathrm{e}$ diversità a tutti i paesaggi europei, si considera "Paesaggio" una parte di territorio così come viene percepita dalle popolazioni, attribuendo a esso valori identitari nazionali e soprattutto locali, poiché il paesaggio è componente essenziale del contesto di vita delle popolazioni, ed è espressione della diversità del loro comune patrimonio culturale e naturale, nonché fondamento della loro identità. Il complesso dei ruderi e delle architetture fortificate della Val Tanaro rappresenta proprio quel patrimonio che risponde al valore di identità e diversità delle popolazioni locali riconosciuto dai documenti internazionali quali la Dichiarazione di Faro.

Poco importa se tali rovine appaiono ai più "inutili" per essere conservate: i ruderi sono "belli" ed è la loro "inutilità" che ne garantisce la bellezza! Una bellezza, un'autenticità che il più delle volte si perde dopo valorizzazioni che trasformano il bene culturale in merce o ancor peggio in location appetibili economicamente. Da questo rischio metteva in guardia già Théophile Gautier nel 1833 quando, nella sua appassionata reazione all'elogio dell'utile per l'utile, rappresentato da una "letteratura prostituita al commercio", definiva l'arte come nobile e autentica "resistenza alla trivialità del presente" (Gautier, 1833). I beni culturali, infatti, sono spesso costretti a "prostituirsi" a causa di esigenze meramente commerciali, senza che Enti preposti alla tutela e Amministrazioni si oppongano, o si impegnino a ridurre gli effetti deleteri di alcune valorizzazioni! Perché ciò avviene? Perché ci consoliamo, convincendoci che questa è l'unica soluzione in tempi di crisi economica? Perché la valorizzazione è utile! Perché da essa deriva un ricco e utilissimo giro d'affari! E allora solo ricominciando ad attribuire anche alle antiche rovine di castelli e fortificazioni valore culturale esse, grazie alle loro trasformazioni e riconfigurazioni, o al loro valore materico, formale, ambientale, potranno tornare a essere utili strumenti affinché si rinnovino le interrelazioni con il territorio e il paesaggio, diventando essi stessi indispensabili punti di accumulazione della memoria dei secoli, degli eventi storici, dei processi economici e di quelli sociali di un popolo o di una società intera. 


\section{Bibliography}

Carbonara, G. (2000). "Restauro archeologico", Costruire in Laterizio, 78, p. 38.

Chavarria Arnau, A. (2015). Detecting and understanding historic landscapes, SAP Società Archeologica, Mantova.

Demeglio, P. (2015). "Insediamenti e archeologia in Alta Val Tanaro: dal transito al presidio", in Devoti, C.; Naretto, M.; Volpiano, M., dr., Studi e ricerche per il sistema territoriale alpino cit., pp. 449.

Dezzi Bardeschi, M. (2017). "Quando la sostanza diventò materia”, in Longhi, A.; Romeo, E., eds., Patrimonio e tutela in Italia. A cinquant'anni dall'istituzione della Commissione Franceschini (1964-1967), Ermes edizioni scientifiche, Roma, pp. 29-39.

Gautier, Th. (1833). "Préface", in Albertus, Paulin, Paris, p. V.

Lambrick, G. (1985). Archaeology and Nature Conservation, Oxford University, Externale Studies.

Ordine, N. (2013). L'utilità dell'inutile, Bompiani, Milano, pp. 8, 86.

Pausania. (2001). Viaggio in Grecia, Guida antiquaria e artística, Rizzo, S., introduzione, traduzione e note, Rizzoli, Milano, Libro VIII, pp. 5-9, 215.

Plinio. (2001). Storia delle arti antiche, Larari, M., intr., Ferri, S., trad., Rizzoli, Naturalis Historia, Milano, Libro XXXVI 2, p. 267.

Romeo, E. (2004). "Il restauro archeologico tra conservazione e innovazione", in Romeo, E., ed., Il monumento e la sua conservazione, Celid, Torino, pp. 101-107.

Romeo, E. (2009). Il "parco archeologico: alcune riflessioni su esperienze internazionali", in Scazzosi, L.; Pelissetti, L.S., eds., Giardini storici a 25 anni dalle carte di Firenze: esperienze e prospettive, Leo S. Olschki Editore, Firenze, pp. 391-402.

Romeo, E. (2012). "Alcune riflessioni sull'utilità dell'essere «rovina» nel paesaggio", in Agribusiness, Paesaggio \& Ambiente, 1, pp. 14-22.

Rudiero, R. (2012-2013). La valorizzazione in progress dei beni archeologici e architettonici: una metodologia, Tesi di Specializzazione, Politecnico di Torino, Torino.

Rudiero, R. (2015). "La conservazione in progress di un insediamento alpino: il caso Santa Giulitta", in Devoti, C.; Naretto, M.; Volpiano, M., eds., Studi e ricerche per il sistema territoriale alpino, ANCSA, Gubbio, pp. 485497.

Scazzosi, L. (2002). "Paesaggio e Archeologia", in Kirova, T., ed., Conservation and restoration of the archaeological heritage, Edizioni AV, Cagliari, pp. 77-81.

Tosco, C. (2007). Il paesaggio come storia, Il Mulino, Bologna.

Woodward, C. (2008). Tra le rovine. Un viaggio attraverso la storia, l'arte e la letteratura, Guanda Editore, Parma, pp. 102-126.

Zevi, B. (1994). Architettura della modernità, Newton, Roma, pp. 66-67. 